\title{
Oedema and vasodilatation in inflammation: the relevance of prostaglandins
}

\author{
T. J. Williams \\ B.Sc.
}

Department of Pharmacology, Institute of Basic Medical Sciences, Royal College of Surgeons of England

\begin{abstract}
Summary
The role of prostaglandins in inflammation is still disputed. Some workers have attempted to fit prostaglandins into a simple model, i.e. inflammatory cell $\rightarrow$ chemical mediator $\rightarrow$ vascular response. Recent work has shown that this approach may be misleading. An alternative model is proposed, involving the interaction of two chemical agents, i.e. a vascular permeability-increasing mediator (such as histamine or bradykinin) and a vasodilator (prostaglandin) which potentiates the plasma exudation produced by the permeability-increasing mediator. The results obtained in testing this model support the further proposal that non-steroidal anti-inflammatory compounds suppress inflammatory swelling by inhibiting vasodilatation.
\end{abstract}

\section{Introduction}

Since the discovery of prostaglandins in inflammatory exudate (Willis, 1969), and that nonsteroidal anti-inflammatory compounds inhibit the synthesis of prostaglandins (Vane, 1971), the mode of action of prostaglandins has been extensively investigated. The observation that injection of prostaglandins into the skin of the guinea-pig and rabbit produces little plasma exudation (Williams and Morley, 1973; Williams, 1976a) is not consistent with a simple mediator concept as has previously been proposed for substances such as histamine and bradykinin. It was found, however, that in guineapig skin, prostaglandins were able to potentiate the exudation produced by vascular permeabilityincreasing mediators (Williams and Morley, 1973). A similar phenomenon was observed in other species (Moncada, Ferreira and Vane, 1973; Thomas and West, 1973).

It was suggested (Williams and Morley, 1973) that prostaglandins may exert their potentiating effect because of their potent vasodilator activity. This hypothesis has been tested using a technique developed to measure plasma exudation and changes in blood flow simultaneously in animal skin (Williams, 1976b). The results of this study suggest a role for prostaglandins in inflammation, and provide an explanation for the mode of action of non-steroidal anti-inflammatory compounds in terms of microvascular changes.

\section{Methods}

Rabbits (New Zealand White, male, 3.5-4.5 kg) received an intravenous injection of methohexitone sodium $(10 \mathrm{mg} / \mathrm{kg})$ followed by intravenous ${ }^{131} \mathrm{I}$ human serum albumin. Multiple injections (36-72) of inflammatory test substances mixed with ${ }^{133} \mathrm{Xe}$ were then given into the dorsal skin (previously clipped). Injections were given in random block order according to a balanced site pattern, with six replicates for each dose. After a predetermined interval (20 or $30 \mathrm{~min})$, the animal was killed, the skin removed, and the injection areas excised with a steel punch (16 $\mathrm{mm}$ diameter). The skin samples were placed in paraffin oil in sealed tubes and counted, together with blood samples and injection fluid samples, in an automatic $\gamma$-counter. Results were computed from punched tape using a PDP-12 computer. Plasma exudation was computed from ${ }^{131}$ I accumulation, and local blood flow changes from ${ }^{133} \mathrm{Xe}$ clearance, as previously described (Williams, 1976b).

\section{Results}

Figure 1 demonstrates that intradermal injection of $\mathrm{PGE}_{2}$ produced a dose-related increase in local blood flow with little evidence of exudation. Figure 2 shows that increasing doses of $\mathrm{PGE}_{2}$ added to a fixed dose of histamine resulted in a marked potentiation of the histamine-induced exudation.

The evidence linking exudation-potentiation with vasodilatation has been previously described (Williams, 1976a). The rank order of potency of different prostaglandins was the same for potentiating and vasodilating activity, i.e. PGE $>$ PGA $>$ PGF $>$ PGD. Other substances which produced an increase in blood flow (although much less potent than prostaglandins), e.g. isoprenaline, adenosine and adenosine diphosphate, also potentiated responses to histamine. Conversely, substances which reduced 

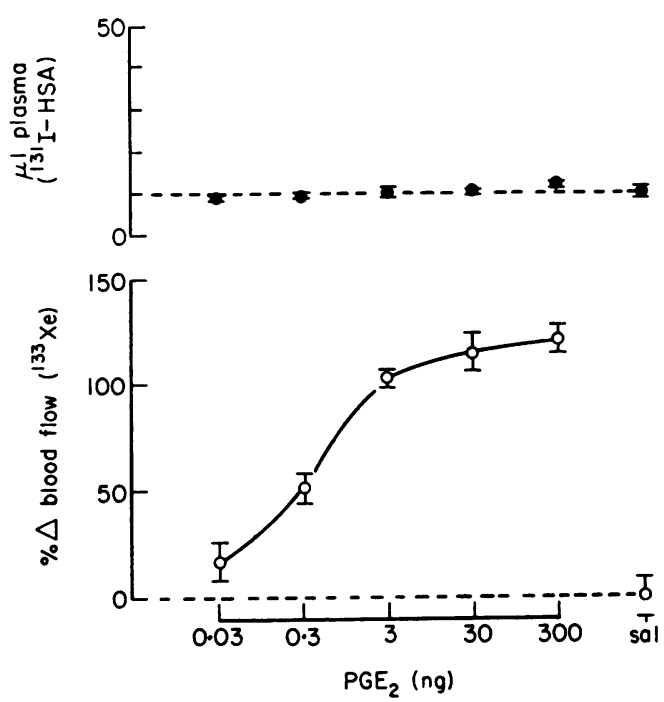

Fig. 1. Dose-response curve to $\mathrm{PGE}_{2}$ in rabbit skin. Top trace shows that increasing doses of $\mathrm{PGE}_{2}$ (in 100 $\mu l$ injections) produced little evidence of exudation, as measured by ${ }^{131}$ I-albumin accumulation. Dotted line represents plasma volume in saline-injected control sites. Lower trace shows increasing blood flow in response to increasing doses of $\mathrm{PGE}_{2}$, as measured by ${ }^{131} \mathrm{Xe}$ clearance. Blood flow is expressed as percentage of flow in saline-injected sites. (Results are expressed as: mean \pm s.e. mean, $n=6$.)

blood flow, e.g. noradrenaline and angiotensin II, reduced responses to histamine. These results strongly suggest that the potentiation of exudation produced by prostaglandins is a result of their vasodilator activity, perhaps as a consequence of increased vessel wall area.

These observations using exogenous agents have been extended to investigate the interaction between permeability-increasing mediators and vasodilator agents in inflammatory reactions produced by injection of foreign materials (Williams, 1977). Intradermal injection of Bordetella pertussis $(2.5 \times$ $10^{8}$ organisms/site) resulted in an increase in blood flow and a small exudation response with a peak at 60-90 min. If the observed exudation resulted from the interaction described, then addition of either exogenous permeability-increasing agent (histamine or bradykinin) or vasodilator $\left(\mathrm{PGE}_{1}\right)$ should potentiate the response. Figure 3 shows that both of these criteria were fulfilled. This experiment is important because it enables one to locate the site of action of a non-steroid anti-inflammatory compound. The non-steroid anti-inflammatory compound, indomethacin, suppressed the potentiation produced by $\boldsymbol{B}$. pertussis and exogenous bradykinin, but failed to suppress the potentiation produced by

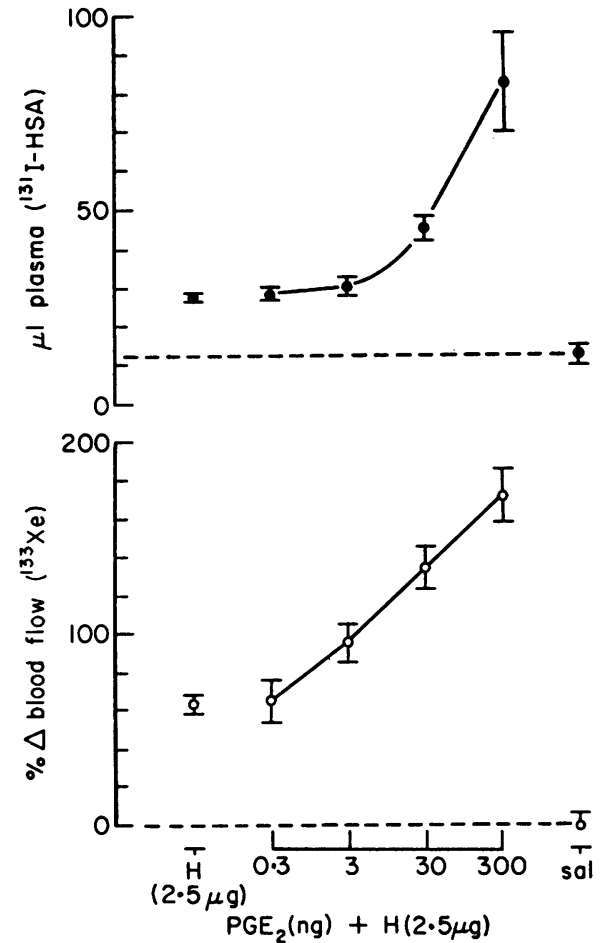

FIG. 2. Potentiation of plasma exudation produced by a fixed dose of histamine $(\mathrm{H})$ and increasing doses of $\mathrm{PGE}_{2}$. (Results expressed as in Fig. 1.)

B. pertussis and exogenous $\mathrm{PGE}_{1}$. This demonstrates that indomethacin suppressed the production of the endogenous potentiating agent (probably an E-type prostaglandin) without affecting the production of the endogenous (unidentified) permeability-increasing mediators.

\section{Discussion}

These results emphasize the importance of prostaglandins (especially PGEs) in inflammation. Their importance would appear to depend on their potent vasodilator activity; this results in a potentiating action on plasma exudation. Because of this phenomenon, concomitantly released permeabilityincreasing mediators may be present in low concentrations in inflammatory reactions.

In the inflammatory model used, indomethacin did not inhibit the production of permeabilityincreasing mediators. This is not consistent with the recently published hypothesis that the permeabilityincreasing mediator is a product of arachidonic acid metabolism, proposed to be $\mathbf{P G G}_{2}$ or a free radical (Kuehl et al., 1977). Further, the frequent observation that non-steroidal anti-inflammatory drugs inhibit mediator release in vitro (e.g. Trethewie, 1951; 


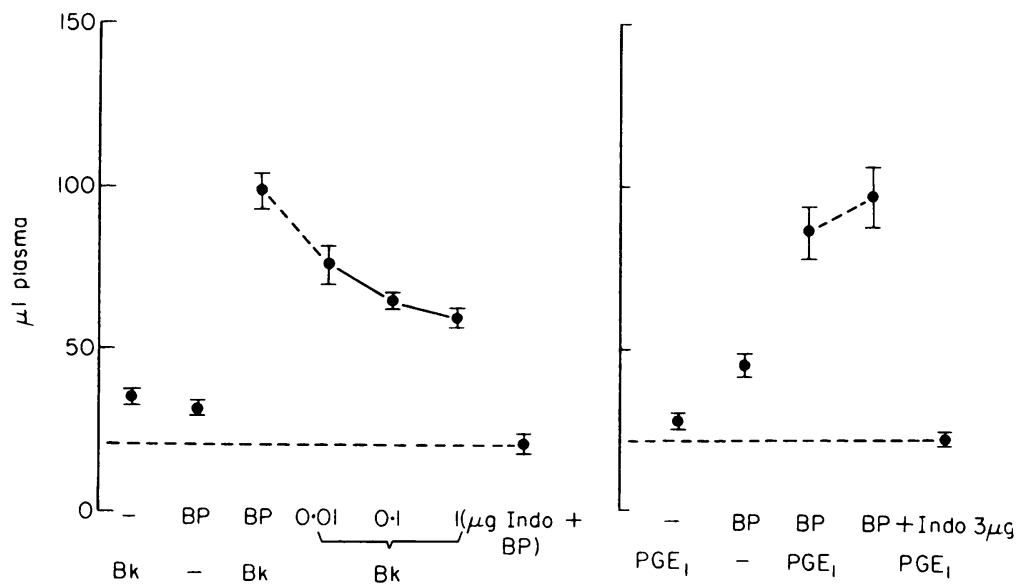

FIG. 3. The effects of indomethacin on the exudation produced by intradermal injection of Bordetella pertussis (BP) in the presence of exogenous bradykinin (Bk, $0.5 \mu \mathrm{g})$ or exogenous $\mathrm{PGE}_{1}(0.5 \mu \mathrm{g})$. Ordinate for both experiments is plasma volume in skin sites. Abscissa: top line is first intradermal injection; lower line is second intradermal injection into the same site 60 min later. Plasma exudation was measured for 30 min following the second injection. Dotted line represents plasma volume in sites given two saline injections, and dashes represent single saline injections. Note that indomethacin (Indo, mixed with $B$. pertussis) suppresses potentiation in $B$. pertussis/bradykinin sites but not the potentiation in $B$. pertussis/PGE 1 sites. (Results are expressed as in Fig. 1.)

Norn, 1971) did not seem to apply to the described in vivo experiments.

These conclusions require verification using other inflammatory test systems, but from the results described, it is proposed that non-steroidal antiinflammatory compounds inhibit inflammatory exudation by suppressing vasodilatation.

\section{References}

Kuehl, F.A., Humes, J.L., Egan, R.W., HaM, E.A., BeveRIDGE, G.C. \& VAN ARMAN, C.G. (1977) Role of prostaglandin endoperoxide $\mathrm{PGG}_{2}$ in inflammatory processes. Nature. London, 265, 170.

Moncada, S., Ferreira, S.H. \& Vane, J.R. (1973) Prostaglandins, aspirin-like drugs and the oedema of inflammation. Nature. London, 246, 217.

NORN, S. (1971) Anaphylactic histamine release and influence of antirheumatics. Acta pharmacologica et toxicologica, 30 (Suppl. 1).

Thomas, G. \& West, G.B. (1973) Prostaglandins as regulators of bradykinin responses. Journal of Pharmacy and Pharmacology, 25, 747.

TrethewiE, E.R. (1951) The influence of sodium salicylate and acetyl salicylic acid on the release of histamine in anaphylaxis. Australian Journal of Experimental Biology and Medical Science, 29, 443.
VANE, J.R. (1971) Inhibition of prostaglandin synthesis as a mechanism of action for aspirin-like drugs. Nature. New Biology, 231, 232.

Williams, T.J. (1976a) The pro-inflammatory activity of EA-, D- and F-type prostaglandins and analogues 16,16dimethyl-PGE ${ }_{2}$ and (15S)-15-methyl-PGE $E_{2}$ in rabbit skin; the relationship between potentiation of plasma exudation and local blood flow changes. British Journal of Pharmacology, 56, 341P.

Williams, T.J. (1976b) Simultaneous measurement of local plasma exudation and blood flow changes induced by intradermal injection of vasoactive substances, using [ $\left.{ }^{131} \mathrm{I}\right]$-albumin and ${ }^{133} \mathrm{Xe}$. Journal of Physiology. London, 254, 4P.

Williams, T.J. (1977) Potentiation of bradykinin-induced exudation following intradermal injection of particulate or colloidal materials in the rabbit: evidence for prostaglandin release and action in inflammation. British Journal of Pharmacology (in press).

Williams, T.J. \& Morley, J. (1973) Prostaglandins as potentiators of increased vascular permeability in inflammation. Nature. London, 246, 215.

Willis, A.L. (1969) Release of histamine, kinin and prostaglandins during carrageenin-induced inflammation in the rat. In: Prostaglandins, Peptides and Amines (Ed. by P. Mantegazza and E. W. Horton), pp. 31-38. Academic Press, London. 